\title{
Importancia del "diagnóstico temprano" en los niños con cáncer para mejorar el pronóstico: concepto con poco sustento científico
}

\author{
Arturo Fajardo-Gutiérrez y Mario Enrique Rendón-Macías \\ Instituto Mexicano del Seguro Social, Centro Médico Nacional Siglo XXI, Hospital de Pediatría, Unidad de Investigación en Epidemiología Clínica, \\ Ciudad de México, México
}

\begin{abstract}
Resumen
La mortalidad por cáncer en niños mexicanos no ha disminuido a los niveles informados en países desarrollados. Una explicación frecuentemente declarada es el alto porcentaje (57.3\%) de pacientes diagnosticados en estadios avanzados (III/IV), atribuible a errores en la sospecha o en la metodología empleada, consideración dudosa si se toma en cuenta que el tiempo de diagnóstico y la proporción de estadios avanzados en México son semejantes a los de países desarrollados. En la mayoría de los niños con cáncer, los días transcurridos desde el primer síntoma a momento del diagnóstico oncológico no correlacionan con el estadio clínico y tampoco con la probabilidad de supervivencia. El éxito en la supervivencia depende en gran medida del tratamiento integral (específico y de la atención a las complicaciones). Esta visión obliga a estrategias dirigidas principalmente a invertir más recursos en opciones terapéuticas eficaces y eficientes, capacitación oncológica integral del equipo de salud (médicos, enfermeras, técnicos), tecnologías diagnósticas, fomento a la colaboración interinstitucional e internacional y apoyo socioeconómico a las familias durante el proceso terapéutico.
\end{abstract}

PALABRAS CLAVE: Cáncer en niños. Tiempo de diagnóstico. Estadio oncológico. Supervivencia oncológica.

\begin{abstract}
Cancer mortality in Mexican children has not decreased to the levels reported in developed countries. A commonly proposed explanation is the high percentage (53.7\%) of patients diagnosed at advanced stages (III/IV), which is attributed to erroneous assumptions or mistakes in the diagnostic approach -a questionable consideration taking into account that both time to diagnosis and the proportion of advanced stage cases in Mexico are similar to those in developed countries. In most cancer cases in children, the number of days elapsed from the moment of the first symptom to the cancer diagnosis is not correlated with clinical stage, and neither with the probability of survival. Survival success largely depends on comprehensive treatment (specific and for the care of complications). This view calls for strategies mainly aimed at spending more resources on efficacious and efficient therapeutic strategies, comprehensive oncology training of healthcare personnel (physicians, nurses and technicians), diagnostic technologies, promotion of interinstitutional and international collaboration and socioeconomic support to families during the therapeutic process.
\end{abstract}

KEY WORDS: Cancer in children. Time to diagnosis. Cancer stage. Cancer survival.

Fecha de recepción: 11-12-2017

Fecha de aceptación: 08-01-2018

DOI://dx.doi.org/10.24875/GMM.18004004
Gac Med Mex. 2018;154:520-526

Disponible en PubMed www.gacetamedicademexico.com 


\section{Introducción}

En 1965, el doctor Alejandro Aguirre, jefe del Servicio de Tumores del Hospital Infantil de México, escribió "Enfermedades malignas de la infancia. Su detección temprana"; en este artículo revisó los principales tumores malignos en niños atendidos en cinco años (19511955). Finalizó su análisis con lo siguiente: ${ }^{1}$

Queremos terminar haciendo un llamado a los pediatras, que forman la avanzada en la lucha por la salud del niño, para que se nos unan en esta cruzada contra el cáncer en la infancia. En esta cruzada de detección temprana de la enfermedad, que es un signo característico universal de esta época de la medicina, desarrollando esta mentalidad anticancerosa, de alerta continua contra estos padecimientos y podríamos decir profiláctica, descubriendo estas neoplasias en su etapa temprana de localización para que la cirugía, radiación y quizás la quimioterapia oportunas puedan prevenir su diseminación metastásica, necesariamente fatal.

Han pasado 52 años y continúa el mismo problema: $57.3 \%$ de los niños atendidos por cáncer (tumores sólidos) en México se diagnostican en estadios avanzados (estadios III/IV). ${ }^{2}$ ¿Qué hemos hecho o dejado de hacer para que después de tanto tiempo este problema perdure? No lo sabemos, pero es un hecho que esto ha justificado el establecimiento de campañas dirigidas a detectar o realizar el diagnóstico en estadios tempranos en niños con cáncer, dirigidas a la población general, médicos generales o familiares y pediatras ${ }^{3}$ aun cuando no existen evidencia científica de su éxito.

En esta revisión narrativa analizaremos la situación de la mortalidad por cáncer en los niños, las peculiaridades del diagnóstico de cáncer en esta población, los estudios que abordan el problema con base en el tiempo al diagnóstico (TDg), la diferencia entre TDg, diagnóstico en etapas tempranas (localizadas) y diagnóstico oportuno (pronóstico) y, finalmente, las implicaciones de estos conceptos en posibles estrategias para lograr la supervivencia con calidad de vida de estos niños.

\section{Mortalidad por cáncer en niños y su tratamiento integral}

La evaluación de la mortalidad por cáncer en la población, tanto en niños como en adultos, cuando no se dispone de otros instrumentos es un buen indicador de la eficacia de un tratamiento integral. ${ }^{4,5}$ Se conoce que la mortalidad por cáncer pediátrico en México no se ha reducido en la misma medida que en los países desarrollados. ${ }^{5}$ En la actualidad, anualmente mueren 57 menores de 15 años por cada millón de niños mexicanos, en contraste con 22 a 30 en países desarrollados. ${ }^{5-7}$ Aunque no se tiene una certeza de la razón de esta diferencia, se han difundido dos aseveraciones no incompatibles:

- La necesidad de incrementar los recursos diagnóstico-terapéuticos oncológicos específicos, aunado a los requeridos para prevenir, mitigar o tratar las complicaciones asociadas. ${ }^{5,6}$

- El retraso en la atención médica oncológica por diagnóstico tardío., ${ }^{1,8}$

Como se mencionó, esta última aseveración ha motivado la implementación de estrategias educativas, dirigidas tanto a la población general como al personal en salud. ${ }^{3}$

\section{Incidencia del cáncer en niños}

Poco se ha reportado sobre la incidencia de cáncer en niños mexicanos. Los datos disponibles han sido informados principalmente por el Instituto Mexicano del Seguro Social y proceden de la Ciudad de México, Estado de México, Morelos, Guerrero y Chiapas. De este registro se ha estimado una incidencia de 128 casos anuales $\times 1000000$ de sujetos $<15$ años, cifra poco menor a 140 estimada mundialmente. ${ }^{9}$ Asimismo, esta incidencia ha sido estable de 2001 a $2013 .^{5}$ En forma específica se espera que aproximadamente $46.2 \%$ de los casos nuevos correspondan a leucemias, $12.1 \%$ a tumores del sistema nervioso, $10.7 \%$ a linfomas y el resto a otros tumores sólidos. ${ }^{5}$ Con estos datos, se calcula que un médico pediatra con una consulta diaria promedio de cinco pacientes $(\approx 1200$ en un año) vería un paciente con cáncer cada seis años..$^{10}$ Feltbower et al. en Inglaterra estimaron que un médico del primer nivel atendería uno o dos niños con cáncer en 20 años. ${ }^{11}$ De tal forma, se debe admitir que el cáncer en niños es raro y, en general, poco probable como causa frecuente de atención médica infantil. Frecuentemente se sospecha cáncer en el niño como consecuencia de un diagnóstico diferencial de alguna enfermedad más común. Por último, se estima que aproximadamente $25 \%$ de los cánceres infantiles será diagnosticado en un servicio de urgencias, sin previa revisión en algún consultorio médico. ${ }^{10}$ 


\section{La sintomatología del cáncer infantil}

Ha sido ampliamente informada la diversidad sintomatológica de los cánceres en pediatría, ${ }^{10-12}$ que pueden semejar cualquier patología, y aunque hay algunos signos y síntomas propios de algunas neoplasias (por ejemplo, leucocoria en retinoblastoma), la mayoría tiene baja especificidad y, por lo tanto, las guías propuestas solo orientan a la sospecha. ${ }^{10,13-16}$ Gran parte de la variabilidad de la sintomatología clínica depende del sitio del tumor, su tamaño y asociación con la funcionalidad del o los órganos involucrados. De esta manera, tumores grandes o accesibles a la exploración física suelen ser más fácilmente detectados. Si afectan la funcionalidad de órganos también darán más sintomatología y, por lo tanto, mayor demanda de atención. Sin embargo, es muy frecuente que algunos tumores se manifiesten con sintomatología inespecífica como cambios en el estado de ánimo o en la conducta, ${ }^{17-19}$ situaciones difícilmente atribuibles en un primer momento a una enfermedad neoplásica.

Aunado a lo anterior, la edad puede tanto limitar como facilitar el diagnóstico, ya que niños muy pequeños en comparación con los mayores muestran menor repertorio signológico. Ante esta inespecificidad de la sintomatología clínica y su alta variabilidad, una premisa propuesta para sospechar un cáncer pediátrico es que todo paciente con uno o varios síntomas persistentes o progresivos debe suscitar su sospecha; ${ }^{15,20}$ la incertidumbre puede traducirse en un dictamen erróneo, incluso en países desarrollados $52 \%$ de los pacientes pediátricos con cáncer tuvieron diagnóstico inicial incorrecto en la primera revisión. ${ }^{13,21}$

\section{Definición del tiempo al diagnóstico}

El TDg ha sido definido como el periodo transcurrido desde que se iniciaron los síntomas asociados con el cáncer hasta su confirmación diagnóstica, ya sea histopatológica o por otras evidencias incontrovertibles (estudio citomorfológico de la médula ósea en niños con leucemias). ${ }^{16,22,23}$

Para fines de análisis y por sus implicaciones en acciones estratégicas para el diagnóstico, el TDg se ha dividido en dos periodos: uno dependiente del paciente (familiares) y otro del sistema de salud (el médico). El primero abarca los días transcurridos desde el momento en que el paciente o su familiar detectan un síntoma asociado con el cáncer hasta la primera consulta médica. El segundo incluye los días desde ese contacto hasta la confirmación diagnóstica. ${ }^{16,22,24}$
Algunos autores han encontrado que en la variabilidad del primer periodo influye la edad del menor, la educación de los padres y el tipo del tumor, ${ }^{24}$ si bien otros no han identificado esta asociación. ${ }^{25}$ En los estudios realizados, el TDg ha sido semejante con medianas que oscilan entre dos y 12.8 semanas; ${ }^{22}$ en el Hospital de Pediatría del Centro Médico Nacional Siglo XX ha sido de cuatro semanas, similar a los informados en países desarrollados; en pacientes colombianos con leucemia aguda, este periodo fue más corto cuando los padres detectaron un síntoma más notorio, como sangrado en piel comparado con palidez (14 versus 40 días). ${ }^{26}$

Para el segundo periodo, dos aspectos han sido considerados fundamentales en su duración:

- El nivel de sospecha por el o los médicos sobre la posibilidad de una neoplasia.

- La disponibilidad o no de métodos útiles para confirmar el diagnóstico. $27-29$

El periodo atribuido a los médicos es de menos días en los países desarrollados, sin embargo, no se analiza el efecto de la falta de perspicacia médica ni de los recursos necesarios para el diagnóstico. ${ }^{29,30}$

Algunos análisis han incluido el tiempo de referencia de las clínicas de primer contacto a los centros de atención oncológica como un factor involucrado. ${ }^{25,31}$ Se ha observado mayor rapidez (< 5 días) en los sistemas de salud mejor integrados, ${ }^{27}$ contrario a aquellos con deficiencias administrativas. ${ }^{27,31}$

En torno al TDg en México, por lo menos en linfomas se determinó que este fue de 20 semanas, ${ }^{24}$ comparado con 7.1 a 14 semanas en Estados Unidos; ${ }^{22,23}$ en particular para los linfomas de Burkitt, el TDg fue menor de 4.5 semanas en el Hospital de Pediatría del Centro Médico Nacional, ${ }^{32} 80 \%$ de los pacientes estaba en estadios III-IV. Según resultados de los pocos estudios realizados, puede concluirse que en México el TDg en niños con cáncer no es muy diferente al determinado en el mundo.

\section{Correlación entre el tiempo al diagnóstico, estadio oncológico y comportamiento biológico de la neoplasia}

En la mente de todos los médicos está realizar un diagnóstico lo más pronto posible por la oportunidad de encontrar un tumor en estadios tempranos 0 , mejor dicho, localizados (I O II). En este sentido, y aunque no lo haga consciente, el médico considera una correlación positiva entre el tiempo de diagnóstico y el estadio clínico: a mayor prolongación del tiempo para 
realizar el diagnóstico oncológico, más avanzado el estadio del tumor al momento de la atención.

Es evidente que un paciente con un tumor localizado y $100 \%$ extirpable tiene más posibilidades de curación, además, los estadios tempranos se asocian con mayor supervivencia, ${ }^{33}$ sin embargo, son pocos los tumores en los que se ha encontrado correlación positiva entre el TDg y el estadio clínico. En un estudio realizado por nuestro grupo solo se encontró correlación positiva en pacientes con retinoblastomas y linfomas de Hodgkin, consistente con los registros en la literatura; ${ }^{34,35}$ en el resto de los grupos de cáncer la correlación fue negativa (mayor tiempo en estadios I-II y menor en estadios III-IV) o no hubo correlación. ${ }^{34}$

Al respecto, Halpering et al. describieron que los meduloblastomas en estadio I-II se diagnosticaron en ocho semanas y que los meduloblastomas en estadios avanzados (III-IV) en menos tiempo (mediana de cuatro semanas), ${ }^{36}$ situación también encontrada por Saha ${ }^{37}$ y Brasme et al. para el sarcoma de Ewing. ${ }^{38}$

En un estudio realizado por nuestro grupo sobre la epidemiología de los linfomas de Hodgkin, en los tumores con histología menos agresiva fue mayor el TDg (26 y 18 semanas en los subtipos histológicos con predominio de linfocitos y esclerosis nodular, respectivamente) y en los de histología más agresiva, más corto (12 y 6.5 semanas para los subtipos histológicos con celularidad mixta y deplesión de linfocitos, respectivamente). ${ }^{39}$ Por ello se sospecha que el TDg tiene mayor correlación con el comportamiento biológico (agresividad del tumor) que con el estadio al diagnóstico. ${ }^{36,40}$

Finalmente, es importante recordar que las leucemias son enfermedades diseminadas dado el involucramiento sanguíneo, por lo tanto, un diagnóstico rápido no implica por naturaleza una neoplasia localizada (I-II). ${ }^{38,40}$

\section{Tiempo al diagnóstico y pronóstico}

En los últimos años se han informado estudios donde se ha analizado si un TDg oncológico corto $(\leq 4$ semanas) influye en el pronóstico de supervivencia de los pacientes pediátricos: ${ }^{18}$ no ha sido posible relacionar el tiempo prolongado con menor supervivencia. Esta observación ya había sido señalada en 2001 por Halpering et al. ${ }^{36}$ y en 2012 por Brasme et al. ${ }^{38}$ Una explicación fue la mayor frecuencia de tumores altamente agresivos con tasas de crecimiento mayor en los pacientes con menos tiempo al diagnóstico. La falta de correlación entre el TDg y la supervivencia ha motivado cuestionar la premisa de que entre más corto el TDg será mejor la supervivencia. ${ }^{35,41,42,43}$ Del mismo modo, en neoplasias diseminadas al diagnóstico como las leucemias, varios autores ${ }^{44-46}$ no encontraron una supervivencia mayor en los pacientes diagnosticados en menor tiempo. El pronóstico se relacionó con el tipo de leucemia y su clasificación de riesgo.

El pronóstico de supervivencia depende del estadio al diagnóstico y de que se ofrezca un tratamiento integral adecuado a los niños con cáncer.

\section{Implicaciones estratégicas del tiempo al diagnóstico}

Estudiar y analizar el TDg oncológico en niños se justifica por las implicaciones sobre acciones o estrategias para acortarlo, y, sobre todo, por la intención de mejorar la supervivencia y calidad de vida. Al respecto, en México se han realizado acciones de difusión sobre los síntomas y signos asociados con el cáncer para el pronto reconocimiento por parte los familiares, así como para la sospecha por parte de los médicos. ${ }^{3}$

En el ámbito internacional se han instaurado cursos de detección temprana del cáncer infantil para médicos familiares y pediatras e, incluso, generación y difusión de guías de práctica clínica para la detección "oportuna", sin que se especifique el concepto de oportunidad, aunque se infiere detección en estadios tempranos. ${ }^{10}$

Finalmente, se han diseñado estrategias para acelerar el envío de pacientes con sospecha de un proceso oncológico, que hasta el momento no han sido evaluadas o no existen reportes al respecto; más aún, se desconoce si han servido para incrementar la supervivencia de los niños con cáncer. De ahí la necesidad de comentar los cuestionamientos que han surgido sobre el impacto real del posible acortamiento del tiempo al diagnóstico en la reducción de la mortalidad de los niños con cáncer; ${ }^{43}$ para lo cual exponemos seis aspectos:

1. En México, el tiempo asociado con los familiares no es sustancialmente diferente al reportado mundialmente. En general, los padres suelen buscar atención médica para su hijo en cuanto detectan algún dato o síntoma. Por otro lado, el cumplimiento de la recomendación sobre la frecuencia de revisiones del niño sano es suficiente para que un médico que explora minuciosamente detecte la presencia de algún tumor. Un sobremensaje a la población puede generar excesivas revisiones y la realización de estudios de laboratorio y 
gabinete innecesarios. La única estrategia reportada como exitosa en la difusión de síntomas de alarma en niños fue una realizada en Honduras, donde se motivó a las madres a observar los ojos de sus bebés, sobre todo en las campañas de vacunación, para detectar leucocoria. Con esta maniobra se logró un diagnóstico de retinoblastomas en estadios más localizados. ${ }^{47}$

2. Cuando un paciente tiene suficiente sintomatología como para pensar en un cáncer (tumor evidente, sangrado importante, fiebre persistente, entre los más comunes), el diagnóstico suele ser rápido. Kundra et al. ${ }^{48}$ registraron $18 \%$ de casos diagnosticados en urgencias y por sintomatología de escasa evolución; en otro informe, la frecuencia fue de $25 \% .^{19}$ El problema son los datos sutiles, que en $90 \%$ de las ocasiones se deben a patología banal y que por cuya persistencia o progresión se sospecha neoplasia. Reino Unido probó una estrategia conocida como "Two strike and go".49 $\mathrm{si}$ el paciente era revisado en más de dos ocasiones por la misma causa y no había mejoría o había agravamiento se daba autorización para una consulta inmediata a un centro oncológico. A los cinco años de la implementación de esta estrategia se incrementaron las consultas innecesarias y la tasa de diagnósticos oncológicos disminuyó, sin que se modificaran los estadios al diagnóstico de los tumores sólidos. Más aún, incluso con errores en el diagnóstico no ha sido posible demostrar que el TDg influya en la supervivencia final. ${ }^{21} \mathrm{~A}$ pesar de ello, se continúa generando guías para facilitar la sospecha diagnóstica, ${ }^{49}$ aun cuando no se ha demostrado que acorten el tiempo de envío o disminuyan los estadios avanzados.

Ante el fracaso de este tipo de estrategias recomendamos hacer el diagnóstico con prontitud si se tienen los recursos para hacerlo, de no ser así es preferible enviar al paciente a un centro oncológico lo más pronto posible. Ello permitirá iniciar un tratamiento en menor tiempo, pero no asegurará que el paciente llegue en un estadio temprano y quizá tampoco un pronóstico favorable.

3. Un problema frecuente en la atención de un niño con sospecha de cáncer es el manejo de la información con los familiares. Tanto no hacer un diagnóstico como informar sobre un cáncer cuando no lo es generará conductas negativas en los familiares (enojo, decepción, angustia, demanda, entre las más frecuentes). ${ }^{50}$ Por ello, recomendamos no ser categóricos hasta no tener evidencia suficiente.

4. En México se ha atribuido la falta de éxito en el aumento de la supervivencia de los niños con cáncer a la alta tasa de casos diagnosticados en estadios avanzados (III-IV). En el Instituto Mexicano del Seguro se han informado casos en estos estadios con variaciones de $31.6 \%$ para retinoblastomas, $67 \%$ para tumores de Wilms y $72 \%$ para linfomas no Hodgkin, ${ }^{2}$ proporciones muy semejante a las informadas en países desarrollados: en Reino Unido se informó $49.5 \%$ de estadios III-V para tumores de Wilms, ${ }^{51}$ en Estados Unidos $36.3 \%$ de estadios III-IV para linfomas de Hodgkin ${ }^{52}$ y en Suiza, Schindler et al. informaron estadio metastático (IV) en $27.7 \%$ de los cánceres infantiles estudiados. ${ }^{53} \mathrm{~A}$ pesar de ello, la supervivencia a cinco años en estos países es alta (> $80 \%$ en Estados Unidos), como consecuencia de la introducción de tratamientos oncológicos altamente eficaces. ${ }^{33}$ Por ello, indicar a los padres que un niño tiene mal pronóstico debido al retraso con el que fue llevado al médico generará sentimientos de culpa injustificados.

5. Las estrategias de referencia inmediata son correctas, sobre todo cuando hay datos clínicos con riesgo de complicaciones irreversibles o muerte. Al respecto, los pacientes con tumores sólidos suelen ser referidos a servicios de urgencias más que aquellos con leucemias, $70 \%$ de los cuales son vistos en un inicio por el médico de primer contacto, ${ }^{54}$ lo cual muestra que existe la posibilidad de realizar estudios antes de enviar a los niños a un servicio oncológico. Por ello insistimos en estudiarlos si se tienen los recursos. Por otro lado, será más eficaz para el tratamiento de estos niños si se invierte en la capacitación a médicos de urgencias sobre el manejo de las complicaciones asociadas con el tratamiento oncológico, como del síndrome febril con neutropenia, ${ }^{55}$ entre otras.

6. Por último, los avances en la supervivencia de los niños con padecimientos oncológicos se deben sobre todo a los tratamientos quimioterápicos, condición no vista hace años cuando el doctor Aguirre lanzó su cruzada. Numerosos esquemas se han apoyado en protocolos estandarizados y controlados en estudios multicéntricos y multinacionales, pero, además, en la inversión de recursos para el apoyo de los pacientes y 
familias durante la aparición de complicaciones inherentes al tratamiento. ${ }^{33}$ De ahí el énfasis en programas de cooperación con centros internacionales, ${ }^{56}$ con los cuales se compartan recursos hospitalarios y capacitación del equipo de salud involucrado en la atención oncológica pediátri$\mathrm{ca}^{57}$ así como continuar con el estudio de los factores de riesgo potencialmente modificables.

\section{Conclusiones}

El éxito en la curación y supervivencia de los niños con cáncer es una realidad y ha sido independiente de la alta tasa de estadios avanzados o diseminados al diagnóstico (particularmente para leucemias y linfomas) y no está relacionado con el TDg. El principal factor responsable ha sido el tratamiento específico antitumoral y el apoyo médico de soporte de las complicaciones. Por ello, aunque es recomendable favorecer un diagnóstico lo más rápido posible, el pilar del éxito es el tratamiento integral, por lo tanto, en México los recursos financieros deberían dirigirse a este en los niños con cáncer.

\section{Bibliografía}

1. Aguirre A. Enfermedades malignas en la infancia. Su detección temprana. Bol Med Hosp Infant Mex 1965;22:235-245.

2. Fajardo-Gutiérrez A, Rendon-Macías ME, Mejía-Aranguré JM. Epidemiología del cáncer en niños mexicanos. Resultados globales. Rev Med Inst Mex Seguro Soc. 2011;49:43-70.

3. Centro Nacional para la Salud de la Infancia y la Adolescencia. [Internet]. Boletín Nacional de Cáncer 2008 a 2012. Disponible en: www.censia. salud.gob.mx. Boletín Nacional de Cáncer 2014.

4. Draper G. Childhood cancer: trends in incidence, survival and mortality. Eur J Cancer. 1995;31:653-654.

5. Fajardo-Gutiérrez A, González-Miranda G, Pachuca-Vázquez A, Allende-López A, Rendon-Macías ME, Fajardo-Yamamoto LM. Cancer incidence and mortality in children in the Mexican Social Security Institute (1996-2013). Salud Publica Mex. 2016;58:162-170.

6. Bosetti C, Bertuccio P, Chatenoud L, Negri E, Levi F, La Vecchia C. Childhood cancer mortality in Europe, 1970-2007. Eur J Cancer. 2010;46:384-394.

7. Chatenoud L, Bertuccio P, Bosetti C, Levi F, Negri E, La Vecchia C. Childhood cancer mortality in America, Asia, and Oceania, 1970 through 2007. Cancer. 2010;116:5063-5074.

8. Rivera-Luna R, Zapata-Tarres M, Shalkow-Klincovstein J, Velasco-Hidalgo L, Olaya-Vargas A, Cárdenas-Cardós $R$, et al. The burden of childhood cancer in Mexico: Implications for low- and middle-income countries. Pediatr Blood Cancer. 2017;64:e26366.

9. Steliarova-Foucher E, Colombet M, Ries LAG, Moreno F, Dolya A, Bray F, et al. International incidence of childhood cancer, 2001-10: a population-based registry study. Lancet Oncol. 2017;18:719-731.

10. Dommett RM, Redaniel T, Stevens MC, Martin RM, Hamilton W. Risk of childhood cancer with symptoms in primary care: a population-based case-control study. Br J Gen Pract. 2013;63:22-29.

11. Feltbower RG, Lewis IJ, Picton S, Richards M, Glaser AW, Kinsey SE, et al. Diagnosing childhood cancer in primary care -a realistic expectation? Br J Cancer. 2004;90(10):1882-1884.

12. Acha-García T. Diagnóstico precoz y signos de alarma en oncohematología pediátrica. En: Asociación Española de Pediatría de Atención Primaria, ed. Curso de actualización en pediatría 2015. España: Lúa Ediciones; 2015

13. Orbach D, Gajdos V, André N. Diagnosis pitfalls and emergencies in children with cancer. Rev Prat. 2014;64:1276-1286.
14. Wilne S, Koller K, Collier J, Kennedy C, Grundy R, Walker D. The diagnosis of brain tumours in children: a guideline to assist healthcare professionals in the assessment of children who may have a brain tumour. Arch Dis Child. 2010;95:534-539.

15. Hamilton W, Hajioff S, Graham J, Schmidt-Hansen M. Suspected cancer (part 1-children and young adults): visual overview of updated NICE guidance. BMJ. 2015;350:h3036-h3036.

16. Lethaby CD, Picton S, Kinsey SE, Phillips R, Van-Laar M, Feltbower RG. A systematic review of time to diagnosis in children and young adults with cancer. Arch Dis Child. 2013;98:349-355.

17. Dixon-Woods M, Findlay M, Young B, Cox H, Heney D. Parents' accounts of obtaining a diagnosis of childhood cancer. Lancet. 2001;357:670-674.

18. Ahrensberg JM, Olesen F, Hansen RP, Schrøder H, Vedsted P. Childhood cancer and factors related to prolonged diagnostic intervals: a Danish population-based study. Br J Cancer. 2013;108:1280-1287.

19. Ahrensberg JM, Fenger-Grøn M, Vedsted P. Primary care use before cancer diagnosis in adolescents and young adults - a nationwide register study. PLoS One. 2016;11:e0155933.

20. Bragonier R, Cordey E. Suspected childhood cancer fast track: increasing referrals, diminishing returns. Arch Dis Child. 2015;100:900-901.

21. Chen J, Mullen CA. Patterns of diagnosis and misdiagnosis in pediatric cancer and relationship to survival. J Pediatr Hematol Oncol. 2017;39:e110-e115.

22. Dang-Tan T, Franco EL. Diagnosis delays in childhood cancer: a review. Cancer. 2007;110:703-713.

23. Pollock BF, Krischer JP, Vietti T. Interval between symptom onset and dianosis of pediaric solid tumors. J Pediatr. 1991;119:725-732.

24. Fajardo-Gutiérrez A, Sandoval-Mex AM, Mejía-Aranguré JM, Rendón-Macías ME, Martínez-García MC. Clinical and social factors that affect the time to diagnosis of Mexican children with cancer. Med Pediatr Oncol. 2002;39:25-31.

25. Vásquez L, Oscanoa M, Tello M, Tapia E, Maza I, Gerónimo J. Factors associated with the latency to diagnosis of childhood cancer in Peru. Pediatr Blood Cancer. 2016;11:1959-1965.

26. Castro-Jimenez MA, Rueda-Arenas E, Cabrera-Rodríguez D. Aproximación a la semiología clínica prediagnóstica, advertida por la madre, de la leucemia linfoide aguda pediátrica. Arch Argent Pediatr. 2015; 113:331-336.

27. Haimi M, Pérez-Nahum M, Stein N, Ben Arush MW. The role of the doctor and the medical system in the diagnostic delay in pediatric malignancies. Cancer Epidemiol. 2011;35:83-89.

28. Brown BJ, James BO, Ajayi SO, Ogun O a, Oladokun RE. Factors influencing time to diagnosis of childhood cancer in Ibadan, Nigeria. Afr Health Sci. 2009;9:247-253.

29. Reaman GH. What, why, and when we image: considerations for diagnostic imaging and clinical research in the Children's Oncology Group. Pediatr Radiol. 2009;39:42-45.

30. Klein-Geltink JE, Pogany LM, Barr RD, Greenberg ML, Mery LS. Waiting times for cancer care in Canadian children: Impact of distance, clinical, and demographic factors. Pediatr Blood Cancer. 2005;44:318-327.

31. Venkatasai JP, Srinivasamaharaj S, Sneha LM, Scott JX, Baby A, Rajan M. Pediatric hematological malignancy: Identification of issues involved in the road to diagnosis. South Asian J Cancer. 2017;6:28-30.

32. Rendón-Macías ME, Valencia-Ramón EA, Fajardo-Gutiérrez A. Clinical and epidemiological characteristics of Burkitt lymphomas in pediatric patients from two defined socioeconomic regions in Mexico. J Trop Pediatr. 2017;63:253-259.

33. Allemani $\mathrm{C}$, Weir HK, Carreira H, Harewood R, Spika D, Wang XS, et al. Global surveillance of cancer survival 1995-2009: analysis of individual data for 25,676,887 patients from 279 population-based registries in 67 countries (CONCORD-2). Lancet. 2015;385:977-1010.

34. Valdez-Ramires L. Tiempo transcurrido entre el inicio de los síntomas y signos, diagnóstico, estadio e inicio del tratamiento en niños con cáncer atendidos en el Instituto Mexicano del Seguro Social. Tesis para obtener el grado de Maestría en Ciencias en Epidemiología, Universidad Nacional Autónoma de México, 2006.

35. Ferrari A, Lo Vullo S, Giardiello D, Veneroni L, Magni C, Clerici CA, et al. The sooner the better? How symptom interval correlates with outcome in children and adolescents with solid tumors: regression tree analysis of the findings of a prospective study. Pediatr Blood Cancer. 2015; 63:479-485.

36. Halperin EC, Watson DM, George SL, Watson DM. Duration of symptoms prior to diagnosis is related inversely to presenting disease stage in children with medulloblastoma. Cancer. 2001;91:1444-1450.

37. Saha V, Love S, Eden T, Micallef-Eynaud P, MacKinlay G. Determinants of symptom interval in childhood cancer. Arch Dis Child. 1993;58:771-774.

38. Brasme JF, Grill J, Doz F, Lacour B, Gaillard S, Delalande O, et al. Long time to diagnosis of medulloblastoma in children is not associated with decreased survival or with worse neurological outcome. PLoS One. 2012;7:e33415. 
39. Rendon-Macías ME, Valencia-Ramón EA, Fajardo-Gutiérrez A, Castro-Ríos A. Incidence of childhood hodgkin lymphoma in Mexico by histologic subtypes and socioeconomic regions. J Pediatr Hematol Oncol. 2015;38:e93-e101.

40. Bacci G, Ferrari S, Longhi A, Forni C, Zavatta M, Smith K, et al. High-grade osteosarcoma of the extremity: differences between localized and metastatic tumors at presentation. J Pediatr Hematol Oncol. 2002;24:27-30.

41. Brasme JF, Gaspar N, Oberlin O, Valteau-Couanet D, Chalumeau M, Grill J. Evidence of increasing mortality with longer time to diagnosis of cancer: is there a paediatric exception? Eur J Cancer. 2014;50:864-866.

42. Neal RD, Tharmanathan P, France B, Din NU, Cotton S, Hendry A, et al. Is increased time to diagnosis and treatment in symptomatic cancer associated with poorer outcomes? Systematic review. Br J Cancer. 2015;112:S92-S107.

43. Kukal K, Dobrovoljac M, Boltshauser E, Ammann RA, Grotzer MA. Does diagnostic delay result in decreased survival in paediatric brain tumours? Eur J Pediatr. 2009;168:303-310.

44. Gupta S, Gibson P, Pole JD, Sutradhar R, Sung L GA. Predictors of diagnostic interval and associations with outcome in acute lymphoblastic leukemia. Pediatr Blood Cancer. 2015;62:957-963.

45. Lins MM, Amorim M, Vitela P, Viana M, Ribeiro RC, Pedrosa A, et al. Delayed diagnosis of leukemia and association with morbid-mortality in children in Pernambuco, Brazil. J Pediatr Hematol Oncol. 2012; 34:e271-e276.

46. Baker JM, To T, Beyene J, Zagorski B, Geenberg ML, Sung L. Influence of length oftime to diagnosis and treatment on the survival of children with acute lymphoblastic leukemia: a population-base study. Leuk Res. 2014;38:204-209

47. Leander C, Fu LC, Peña A, Hoard SC, Rodríguez-Galindo C, Wilimas JA et al. Impact of an education program on late diagnosis of retinoblastoma in Honduras. Pediatr Blood Cancer. 2007;49:817-819.
48. Kundra M, Stankovic C, Gupta N, Thomas R, Hamre M, Mahajan P. Epidemiologic findings of cancer detected in a Peditric Emergency Department. Clin Peditr. 2008;48:404-409.

49. Bragonier R, Cordey E. Suspected childhood cancer fast track: increasing referrals, dismishing returns. Arch Dis Child. 2015;100:900-901.

50. Mu PF, Lee MY, Shen CC, Tung PC, Huang LY, CYW. The experiences of family members in the year following he diagnosis of a child or adolescent with cancer: a qualitative systematic review. JBI Database System Rev Implement Rep. 2015;13:293-329.

51. Fawkner-Corbett DW, Howell L, Pizar BL, Dominici C, McDowell HP, Losty P. Wilms' tumor-lessons and outcomes-a 25-year single center UK experience. Peditric Hematol Oncol. 2014;31:400-408.

52. Schwartz CL, Chen L, McCarten K, Wolden S, Constine LS, Keller FG, et al. Childhood Hodgkin International Prognostic Score (CHIPS) predicts event-free survival in Hodgkin lymphoma: a report from the Children's Oncology Group. Pediatr Blood Cancer. 2017;64:e26278.

53. Schindler M, Belle FN, Grotzer MA, Von-Der-Weid NX, Kuehni CE. Childhood cancer survival in Switzerland (1976-2013): time-trends and predictors. Int J Cancer. 2017:140:62-74.

54. Roskin J, Diviney J, Nanduri V. Presentation of childhood cancers to a paediatric shared care unit. Arch Dis Child. 2015;100:1131-1135.

55. Spencer S, Nypaver M, Hebert K, Benner C, Stanley R, Cohen D, et al. Successful emergency department interventions that reduce time to antibiotics in febrile pediatric cancer patients. BMJ Qual Improv Rep. 2017;6:u212406.w4933.

56. Ribeiro RC, Antillon F, Pedrosa F, Pui CH. Global pediatric oncology: lessons from partnerships between high-income countries and low-to mid-income countries. J Clin Oncol. 2016;34:53-61.

57. Shulman LN, Wagner CM, Barr R, Lopes G, Longo G, Robertson J, et al. Proposing essential medicines to treat cancer: methodologies, processes, and outcomes. J Clin Oncol. 2016;34:69-75 